\title{
Synthetic miR-34a Mimics as a Novel Therapeutic Agent for Multiple Myeloma: In Vitro and In Vivo Evidence
}

Maria T. Di Martino ${ }^{1}$, Emanuela Leone ${ }^{1}$, Nicola Amodio ${ }^{1}$, Umberto Foresta ${ }^{1}$, Marta Lionetti ${ }^{3}$, Maria R. Pitari ${ }^{1}$, Maria E. Gallo Cantafio ${ }^{1}$, Annamaria Gullà ${ }^{1}$, Francesco Conforti ${ }^{2}$, Eugenio Morelli ${ }^{1}$, Vera Tomaino ${ }^{1}$, Marco Rossi $^{1}$, Massimo Negrini ${ }^{4}$, Manlio Ferrarini ${ }^{5}$, Michele Caraglia ${ }^{6}$, Masood A. Shammas ${ }^{7,8}$, Nikhil C. Munshi ${ }^{7,8}$, Kenneth C. Anderson ${ }^{7}$, Antonino $\mathrm{Neri}^{3}$, Pierosandro Tagliaferri ${ }^{1}$, and Pierfrancesco Tassone ${ }^{1,9}$

\section{Abstract}

Purpose: Deregulated expression of miRNAs has been shown in multiple myeloma (MM). A promising strategy to achieve a therapeutic effect by targeting the miRNA regulatory network is to enforce the expression of miRNAs that act as tumor suppressor genes, such as miR-34a.

Experimental Design: Here, we investigated the therapeutic potential of synthetic miR-34a against human MM cells in vitro and in vivo.

Results: Either transient expression of miR-34a synthetic mimics or lentivirus-based miR-34a-stable enforced expression triggered growth inhibition and apoptosis in MM cells in vitro. Synthetic miR-34a downregulated canonic targets BCL2, CDK6, and NOTCH1 at both the mRNA and protein level. Lentiviral vector-transduced $\mathrm{MM}$ xenografts with constitutive miR-34a expression showed high growth inhibition in severe combined immunodeficient (SCID) mice. The anti-MM activity of lipidic-formulated miR-34a was further shown in vivo in two different experimental settings: (i) SCID mice bearing nontransduced MM xenografts; and (ii) SCID-synth-hu mice implanted with synthetic 3-dimensional scaffolds reconstituted with human bone marrow stromal cells and then engrafted with human MM cells. Relevant tumor growth inhibition and survival improvement were observed in mice bearing TP53-mutated MM xenografts treated with miR-34a mimics in the absence of systemic toxicity.

Conclusions: Our findings provide a proof-of-principle that formulated synthetic miR-34a has therapeutic activity in preclinical models and support a framework for development of miR-34a-based treatment strategies in MM patients. Clin Cancer Res; 18(22); 6260-70. (C2012 AACR.

\section{Introduction}

Multiple myeloma (MM) is the second most common hematologic malignancy in Western countries. Despite advances in the understanding of MM pathobiology and

Authors' Affiliations: ${ }^{1}$ Department of Experimental and Clinical Medicine, Magna Graecia University and Medical Oncology Unit, T. Campanella Cancer Center, Salvatore Venuta University Campus; '2Pathology Unit, Magna Graecia University, Catanzaro, Italy; ${ }^{3}$ Department of Medical Sciences University of Milan, Hematology 1, IRCCS Policlinico Foundation, Milan, Italy; ${ }^{4}$ Department of Experimental and Diagnostic Medicine, Interdepartmental Center for Cancer Research, University of Ferrara, Ferrara, Italy; ${ }^{5}$ Division of Medical Oncology C, Istituto Nazionale per la Ricerca sul Cancro and Department of Internal Medicine, University of Genoa, Genoa, Italy; ${ }^{6}$ Department of Biochemistry and Biophysics, Second University of Naples, Naples, Italy; ${ }^{7}$ Department of Medical Oncology, Dana-Farber Cancer Institute; ${ }^{8}$ Boston Veterans Administration Healthcare System, West Roxbury, Boston, Massachusetts; and ${ }^{9}$ Temple University's College of Science \& Technology, Philadelphia, Pennsylvania

Note: Supplementary data for this article are available at Clinical Cancer Research Online (http://clincancerres.aacrjournals.org/).

Corresponding Author: Pierfrancesco Tassone, Magna Graecia University, Viale Europa, 88100 Catanzaro, Italy. Phone: 39-0961-3697029; Fax: 39-0961-3697341; E-mail: tassone@unicz.it.

doi: 10.1158/1078-0432.CCR-12-1708

(C)2012 American Association for Cancer Research. development of novel therapeutic strategies, available treatments fail to cure the disease in most cases (1-3). A variety of genetic and epigenetic abnormalities characterizes the MM multistep transformation process occurring in the bone marrow (BM), where the $\mathrm{BM}$ microenvironment (BMM) plays a key supportive role for growth, survival, and drug resistance of tumor cells $(1,4-6)$. All these alterations can dramatically deregulate the plasma cell growth and the network of molecular interactions within the human BMM.

miRNAs are small noncoding RNAs of 19 to $25 \mathrm{nt}$ that play a critical role in the posttranscriptional regulation of gene expression $(7,8)$. As a result of imperfect pairing with target mRNAs, they may cause repression of translation or degradation of mRNAs. These small molecules are important modulators of key regulatory cellular pathways and may play a relevant role in tumorigenesis (9), as deregulated miRNAs can act as oncogenes (Onco-miRNAs) or tumorsuppressors (TS-miRNAs; refs. 10, 11). Therefore, miRNAs have elicited a growing interest in the cancer research community as new potential tumor cell targets (OncomiRNAs) or as new potential anticancer agents (TS-miRNAs), because of their ability to target multiple genes in the context of signaling networks involved in cancer promotion or repression (12). Specifically, miRNAs are variably 


\section{Translational Relevance}

The miRNA regulatory network is emerging as a novel target for the treatment of human cancer. In this study, we investigated the therapeutic potential of synthetic miR-34a against TP53-mutated human multiple myeloma (MM) cells in vitro and in vivo. The translational relevance of our study resides in the findings that intratumor or systemic delivery of novel lipidic-formulated synthetic miR-34a induces antimyeloma activity in vivo in different murine models of human $\mathrm{MM}$, including the most innovative SCID-synth-hu system, in the absence of systemic toxicity in treated animals. Moreover, the specificity of the miR-34a in vivo activity was shown by selective downregulation of canonic targets BCL2 and CDK6. Taken together, our results indicate that formulated synthetic miR-34a is an active agent against MM, which merits further investigation for clinical development in this still incurable disease.

involved in the pathogenesis of human MM and several miRNAs have been found to be abnormally upregulated or downregulated in primary MM cells or cell lines, as recently reviewed (13-16). Although the biologic role of miRNAs in the pathogenesis of $\mathrm{MM}$ is presently well documented by several studies, only few reports support the notion that miRNAs have a potential in MM therapy (16-18). For example, Roccaro and colleagues (17) showed a downregulation of $15 \mathrm{a} / 16$ miRNAs in MM. Because these miRNAs appear to be negative regulators of MM cell proliferation by inhibiting AKT3, ribosomal protein S6, MAP kinases, and NF-kappaB activator MAP3KIP3, it was suggested that the reconstitution of normal miRNA expression could represent a MM treatment. More recently, Pichiorri and colleagues (18) showed a MM-specific miRNA signature characterized by miR-192, 194, and 215 downregulation in a subset of newly diagnosed MMs. These miRNAs are transcriptionally activated by TP53 and are also positive regulators of TP53, thereby producing a loop of major relevance in the biology of $\mathrm{MM}$, which can be a potential target for therapeutic intervention.

Among miRNAs frequently deregulated in human cancer, miR-34a is of special interest in the field of miRNA therapeutics (19-21). Specifically, hypermethylation of miR-34a promoter has been found in MM patient cells and MM cell lines, but not in normal counterparts (22). miR-34a, first described as potential TS-miRNA (23), belongs to a miRNA family including miR-34b and miR-34c and is encoded by a gene located on $1 \mathrm{p} 36$ (21). miR-34a transcription is induced by TP53 in response to cell stress, thereby promoting apoptosis, cell-cycle arrest, and senescence (24-29). Recent reports show that induction of miR-34a expression could overcome TP53 loss of function in pancreatic cancer cells $(22,30)$. Although some of these events may, at least in part, be related to the positive feedback loop that links miR34a to TP53 (31), some reports suggest that the antitumor activity of miR-34a might be independent of TP53 mutational status $(32,33)$. Moreover, in one report miR-34a antitumor activity is not limited to cell lines with reduced endogenous miR-34a expression levels, but it is also effective in cells with an apparently normal miRNA expression (33).

In this study, we investigated a novel anti-MM therapeutic strategy based on miR-34a. The main challenges for an effective miRNA-based therapy include the effective delivery of the appropriate miRNA to and its uptake by malignant plasma cells in their specific microenvironment without offtarget effects. Therefore, we characterized the in vitro and in vivo anti-MM activity and molecular perturbations produced by synthetic miR-34a mimics. For in vivo studies, synthetic miR-34a mimics were formulated in a novel neutral lipid emulsion (NLE; refs. 33, 34) delivery system and, to explore the clinical translatability of experimental findings, we examined the antitumor activity in murine xenograft models of human MM. Our findings support the development of formulated miR-34a as an experimental new agent for the treatment of MM.

\section{Materials and Methods}

\section{Cell lines and primary cultures}

NCI-H929, U266, and SKMM1 MM cell lines were available within our research network, although the RPMI-8226 $\mathrm{MM}$ cell line was purchased from Istituto Zooprofilattico Sperimentale (I.Z.S.L.E.R.). OPM1, DOX-6, and LR-5 MM cell lines were kindly provided by Dr. Eduard Thomson (University of Texas Medical Branch, Galveston, TX, USA), MM1S was purchased by American Type Culture Collection, INA-6 was provided by Dr. Renate Burger (University of Erlangen-Nuernberg, Erlangen, Germany; refs. 35, 36). Human BM stromal cells (BMSC) were obtained by longterm culture of $\mathrm{BM}$ mononuclear cells, as previously described (37-39). Primary CD138+ patient MM cells were obtained by Ficoll gradient separation followed by positive selection from patient BM aspirates, using CD138 MicroBeads antibody (MACS, Milteny Biotec). For coculture, $1 \times$ $10^{5}$ CD138+ cells were seeded on $5 \times 10^{4}$ BMSCs, which had been cultured for 24 to 48 hours in 96-well plates.

In vitro transfection of MM cells with synthetic miR-34a

Synthetic pre-miRNAs were purchased from Ambion (Applied Biosystems). A total of $1 \times 10^{6}$ cells were electroporated with scrambled (miR-NC) or synthetic pre-miR$34 \mathrm{a}$ (miR-34a) at a final concentration of $100 \mathrm{nmol} / \mathrm{L}$, using Neon Transfection System (Invitrogen) with 1050 V, 30 ms, 1 pulse. Cell transfection efficiency was evaluated by flow cytometric analysis of FAM-dye-labeled synthetic miRNA inhibitor (Invitrogen) transfection.

\section{Quantitative real-time amplification of miRNAs and mRNAs}

Total RNA from MM cells was prepared with the TRIzol Reagent (Invitrogen) according to manufacturer's instructions. Oligo-dT-primed cDNA was obtained using the High Capacity cDNA Reverse Transcription Kit (Applied 
Biosystems). The single-tube TaqMan miRNA assays were used to detect and quantify mature miR-34a and target mRNAs, according to the manufacturer's instructions by the use of the StepOne Thermocycler and the sequence detection system (Applied Biosystems). miR-34a and mRNAs were normalized on RNU44 (40) and glyceraldehyde-3-phosphate dehydrogenase (GAPDH; Ambion), respectively. Comparative real-time PCR (RT-PCR) was conducted in triplicate, including no-template controls. Relative expression was calculated using the comparative cross-threshold (Ct) method (41).

\section{Apoptosis analysis \\ MM cells transfected or transduced with miR-34a or scrambled sequence/empty vector were harvested and trea- ted with Annexin V/7-aminoactinomycin D (7-AAD) solu- tion (BD Pharmingen) at 24, 48, and 72 hours, according to the manufacturer's instructions.}

\section{Animals and in vivo models of human MM}

Male CB-17 severe combined immunodeficient (SCID) mice ( 6 to 8 weeks old; Harlan Laboratories, Inc.) were housed and monitored in our Animal Research Facility. All experimental procedures and protocols had been approved by the Institutional Ethical Committee (Magna Graecia University) and conducted according to protocols approved by the National Directorate of Veterinary Services (Italy). In accordance with institutional guidelines, mice were sacrificed when their tumors reached $2 \mathrm{~cm}$ in diameter or in the event of paralysis or major compromise in their quality of life, to prevent unnecessary suffering. For our study, we use 3 different models of human MM, including (i) SCID mice bearing lentiviral vector-transduced MM xenografts; (ii) SCID mice bearing subcutaneous MM xenografts (42); and (iii) SCID mice implanted with a 3-dimensional polymeric scaffold previously reconstituted with human BMSCs and then injected with human MM cells (SCID-synth-hu; refs. 43-45)

\section{Statistical analysis}

Student $t$ test, 2-tailed, and Log rank test were used to calculate all reported $P$ values using GraphPad software (www.graphpad.com). Graphs were obtained using SigmaPlot version 11.0.

\section{Results}

\section{Expression of miR-34a in MM cells}

We first evaluated the miR-34a expression in a series of 11 MM cell lines by quantitative RT-PCR (q-RT-PCR; for details about MM cell lines, see Supplementary Methods). Among these, 2 wild-type TP53 cell lines (MM1S and NCI-H929) showed significantly higher miR-34a expression as compared with TP53-mutated MM cells (U266, SKMM1, RPMI8226, INA-6, DOX6, KMS12-PE, OPM1, OPM2, and LR5; Fig. 1A). We next explored if the positive miR-34aTP53 loop was functional in MM cells. Specifically, we treated the TP53 mutated SKMM1 and RPMI-8226 cells, as well as the TP53 wild-type NCI-H929 cell line with nutlin-3, which blocks the TP53-MDM2 inhibitory interaction and thereby induces the expression of TP53-regulated genes (46). As expected, nutlin-3 treatment induced miR-34a expression in TP53 wild-type cells (NCI-H929) but not in TP53-mutated SKMM1 and RPMI-8226 cells, as evaluated by q-RT-PCR $(P<0.05$, Fig. 1B). Nutlin-3-induced upregulation of miR-34a in turn reduced expression of miR-34a canonical targets, such as BCL2 and CDK6 proteins (for Western blotting procedures see Supplementary Methods). As expected, this effect was not demonstrable in TP53mutated RPMI-8226 cells (Fig. 1C). These findings suggest that miR-34a expression is positively modulated by a functional loop in TP53 wild-type MM cells. An additional finding reinforcing the role of TP53 in regulation of miR34 a expression is the significantly downregulated expression of miR-34a in a microarray data set of TP53-mutated CD138+ primary MM cells as compared with TP53-wildtype MM cells (47).

\section{In vitro enforced expression of miR-34a in MM cells}

To evaluate the biologic effects of miR-34a, we transfected low-miR-34a MM cell lines with synthetic miR-34a or miRNC by electroporation. The transfection efficiency was $98 \%$, as determined by FAM-dye-labeled oligonucleotide transfection and subsequent flow cytometric analysis (Supplementary Fig. S1A). In a parallel experiment, the biologic activity of transfected synthetic miRNA was assessed by downregulation of PTK9 mRNA induced by miR-1 (Supplementary Fig. S1B). The antiproliferative effect induced by miR-34a in MM cells was evaluated by Trypan Blue exclusion assay (procedure available in Supplementary Methods) after synthetic miR-34a or miR-NC transfection. We observed a significant growth inhibition in TP53-mutated SKMM1 $(P<0.005$, Fig. 2A), RPMI-8226 ( $P<0.05$, Fig. 2B), and OPM1 $(P<0.005$, Fig. 2C) MM cells. On the other hand TP53-wild type MM1S and NCI-H929 cells, where a functional TP53-miR34a loop is operative, were not inhibited by miR-34a (Supplementary Fig. S2), although transfection of anti-miR-34a oligonucleotides indeed produced a growth stimulus in these cells (Fig. 2D and E). These data further confirm miR-34a role as a negative regulator of MM cell growth in TP53-wild type cells, and strengthen the rationale of our experimental strategy of enforced expression in TP53mutated MM cells.

We next investigated the induction of apoptosis by Annexin V/7-AAD assay. An increase of apoptotic cell death was observed in TP53-mutated cells following transfection with miR-34a, but not with miR-NC, after 48 hours (Fig. 2F and G) that was more evident in SKMM1 cells. In contrast, this effect was not observed in TP53 wild-type cells (data not shown). To further explore the anti-MM effects induced by miR-34a, we carried out a clonogenic assay to study the colony formation activity of transiently transfected cells. We found a $45 \%$ and 20\% reduced SKMM1 and RPMI-8226 colony formation, respectively, 14 days after transfection (Fig. 2H). These findings indicate that miR-34a inhibits clonogenic properties of MM cells. 


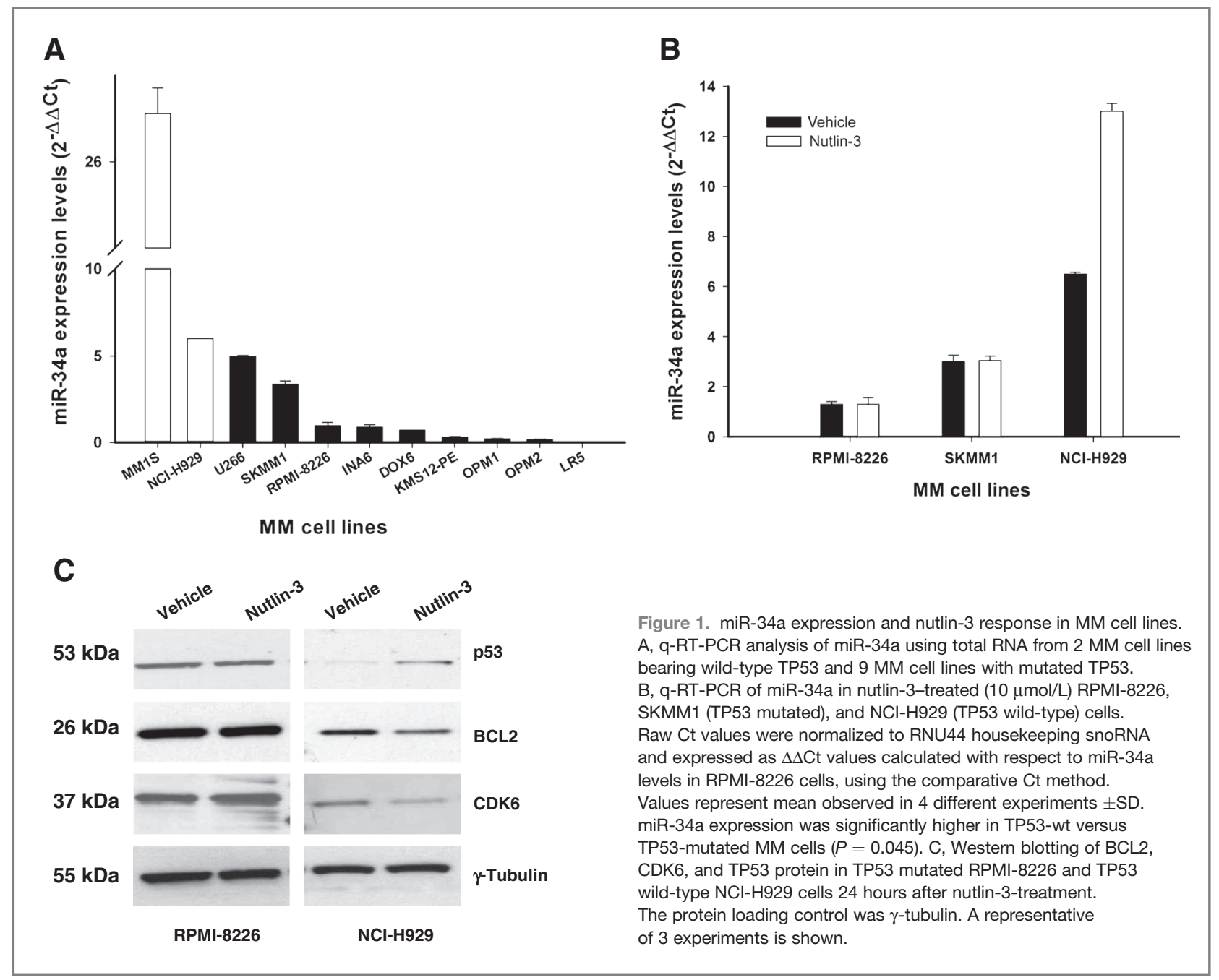

In vitro effect of $\mathrm{MM}$ cell transduction with lentiviral miR-34a expression vector

To achieve stable expression of miR-34a, the feline immunodeficiency lentiviral miR-34a expression vector was used to infect MM cells (for details see Supplementary Methods). About 97\% transduction efficiency was shown by flow cytometric GFP analysis (Supplementary Fig. S3). As shown in Supplementary Fig. S3A, the stable expression of miR-34a affected proliferation of SKMM1 cells in a time-dependent manner, as showed by MTS assay. Consistently, we detected a 2 -fold increase of early and late apoptosis 48 hours after transduction with miR34a, as evidenced by Annexin/7-AAD assay (Supplementary Fig. S4B). Moreover, the miR-34a expression levels, analyzed by q-RT-PCR after lentivirus infection, showed 3-fold increase in pMIF-34a compared with pMIF empty-vector transduced cells (Supplementary Fig. S4C). These findings showed that lentivirus transduction of miR-34a results in a significant anti-MM effect in vitro.

\section{Downregulation of validated miR-34a targets in MM cell lines.}

To investigate whether genes known to be regulated by miR-34a were modulated by exogenous synthetic mimics, we analyzed BCL2, CDK6, and NOTCH1 mRNA levels by qRT-PCR in miR-34a-transfected SKMM1 and RPMI-8226 cells. As shown in Fig. 3A and B, we detected a significant downregulation of CDK6 and NOTCH1 mRNA expression 24 hours after cell transfection. This effect occurred together with downmodulation of CDK6 and BCL2 proteins evaluated by Western blot analysis (Fig. 3C and D). Altogether, these results show that synthetic miR-34a activity modulates validated targets.

\section{miR-34a lentiviral transduction inhibits MM xenograft formation in SCID mice}

In the in vivo studies, we examined the effect of transduced miR-34a on the tumorigenic potential of TP53-mutated MM cells engrafted in SCID mice (for in vivo experiments see Supplementary Methods). As shown in Fig. 4A, 


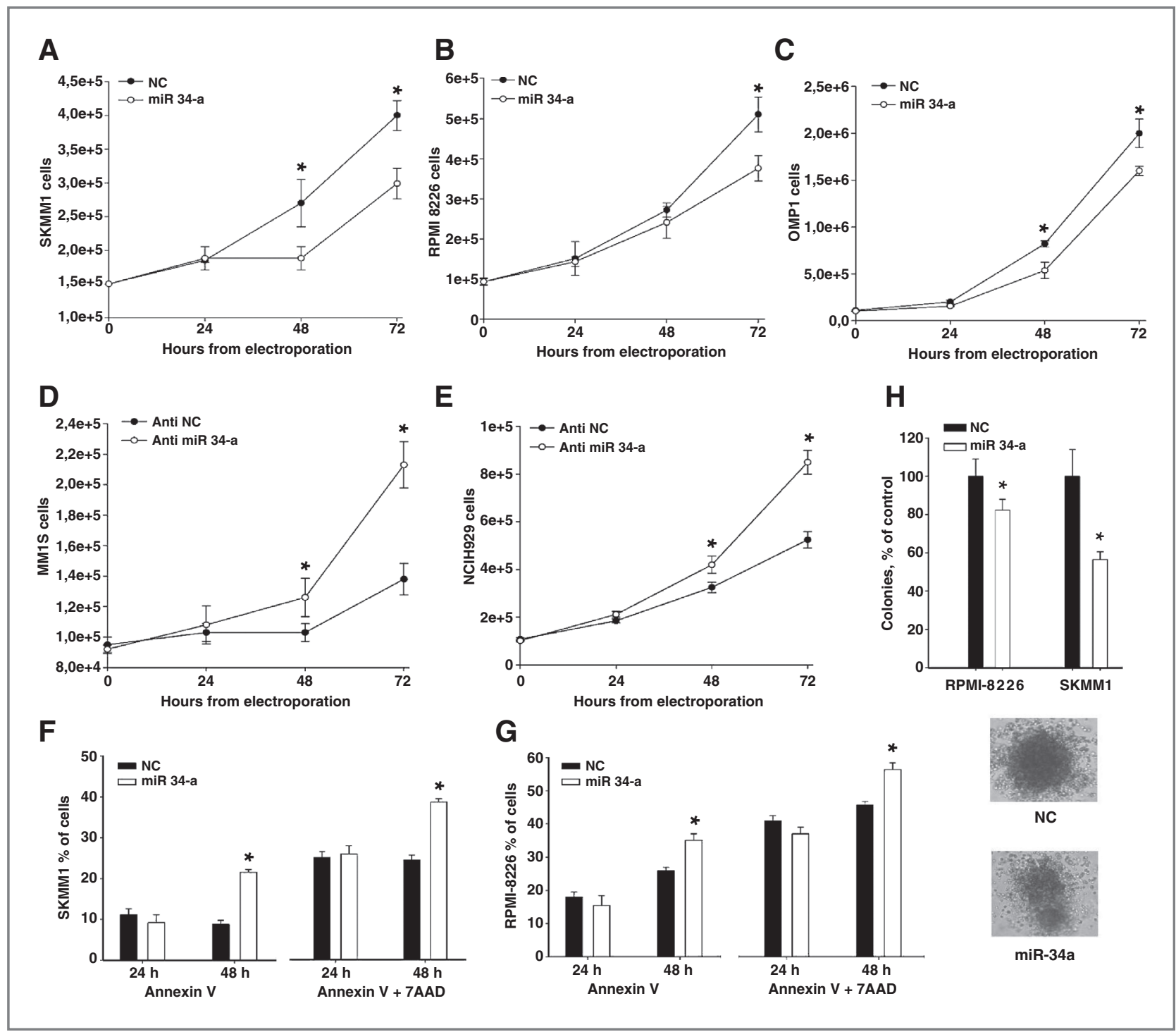

Figure 2. miR-34a has antiproliferative activity and induces apoptosis in MM cell lines. Cell growth analysis of SKMM1 (A), RPMI-8226 (B), or OPM1 (C) cells transfected with miR-34a or miR-NC oligonucleotide control. Average \pm SD values of 3 independent experiments are plotted. $P$ values calculated by Student $t$ test, 2 -tailed, at 48 and 72 hours, respectively, after transfection, are: 0.01 and 0.005 for SKMM1; 0.001 and 0.05 for RPMI-8226; 0.006 and 0.008 for OPM1. Significant $P$ values $(P<0.05)$ are indicated by *. Cell growth analysis of MM1S (D) and NCl-H929 (E) cells transfected with miR-34a inhibitor (anti-miR-34a, Life Technologies AM11030) and anti-miR-scrambled inhibitor (NC, Life Technologies AM17010) oligonucleotide control. Average \pm SD values of 3 independent experiments are plotted. $P$ values calculated by Student $t$ test, 2-tailed, were 0.04 and 0.002 for MM1S cells at 48 and 72 hours after transfection; and $0.04,0.01$, and 0.0008 for NCl-H929 cells at 24, 48, and 72 hours after transfection. Annexin V/7-AAD analysis of SKMM1 (F) and RPMI-8226 (G) cells after transfection with synthetic anti-miR-34a or control. Results are shown as percentage of apoptotic cells. Data are the average \pm SD of 3 independent experiments. $\mathrm{H}$, colony formation assay using RPMI-8226 and SKMM1 cells. Cells were transfected with synthetic miR-34a or miR-NC by electroporation in triplicate, and then seeded at 2,000 cells per 18-well plate in methyl cellulosebased medium. After 2 weeks, colony formation capacity was evaluated by counting colonies including $>100$ cells. Means \pm SE for 3 independent experiments are indicated. In all the experiments, $P$ value was $<0.03$ comparing miR-34a versus NC. A representative image of miR-34a and NC SKMM1 colonies shows homogeneous features of colonies formed by transfected cells with miR-NC, whereas cells transfected with miR-34a form irregular heterogeneous colonies.

enhanced expression of miR-34a caused a significant inhibition $(P<0.05)$ of tumor formation. In addition, the average size of miR-34a transduced tumors was significantly $(P=0.008)$ lower compared with control group. Quantitative analysis of miR-34a levels in retrieved tumors confirmed a more than 3-fold increase in pMIF-34a-transduced tumors (Supplementary Fig. S5A). Histologic and immu- nohistochemical analysis of excised pMIF-34a tumors disclosed large areas of necrosis with abundant nuclear debris ("dust-like" nuclear fragments, Fig. 4B). Moreover, few viable cells at the tumor periphery exhibited cleaved caspase-3 and lower expression of Ki-67, indicating that miR34 a expression inhibits proliferation and stimulates the apoptotic cascade in SKMM1 xenografts. Analysis of 
Figure 3. Molecular effects induced by transient expression of miR-34a in MM cells. q-RT-PCR of BCL2, CDK6, and NOTCH1 after transfection with synthetic miR-34a or miR-NC in SKMM1 (A) and RPMI8226 (B) cells. The results are shown as average mRNA expression after normalization with GAPDH and $\Delta \triangle \mathrm{Ct}$ calculations. Data represent the average $\pm S D$ of 3 independent experiments. Western blotting of BCL2 and CDK6 protein in SKMM1 (C) and RPMI-8226 (D) cells 24 and 48 hours after transfection with synthetic miR-34a or scrambled oligonucleotides (NC). The protein loading control was $\gamma$-tubulin. Experiments were conducted in triplicate. miR-34a effects on protein levels reached statistical significance $(P<0.05)$ at all time points.
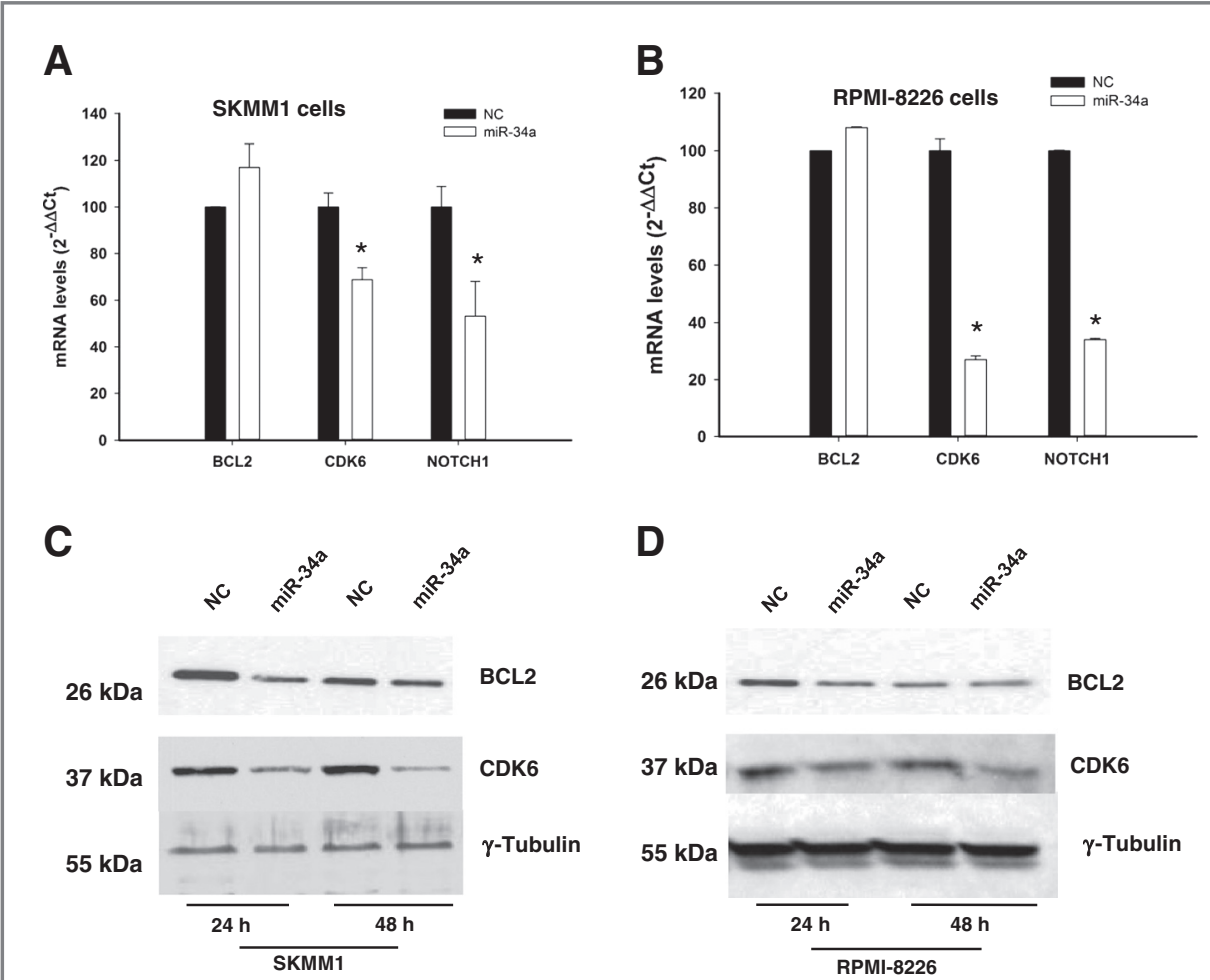

D

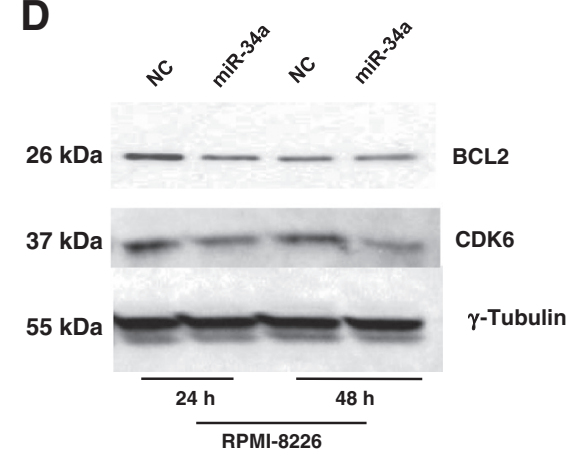

miR-34a targets at mRNA and protein levels showed that lentiviral-mediated ectopic expression of miR-34a induced BCL2, CDK6, and NOTCH1 downregulation in pMIF-34atransduced tumors (Fig. 4C). Although BCL2 mRNA was downregulated in retrieved tumors, electroporation of miR-34a was unable to produce the same effect in vitro (Fig. 3). On the basis of these findings, we suggest that long-term exposure as in constitutive in vivo xenografts is required to reduce BCL2 mRNA level, although a shortterm exposure is sufficient to impair BCL2 protein synthesis. All together, these findings indicate miR-34a-dependent regulation of canonical targets BCL2, CDK6, and NOTCH1 in vivo.

\section{Inhibition of MM xenografts in SCID mice by intratumoral delivery of formulated miR-34a}

We next investigated the effect of miR-34a treatment on MM xenograft growth in SCID mice. miR-34a or miR-NC were administered with NLE particles, a formulation specifically designed for systemic delivery of oligonucleotides in vivo $(33,34)$. A highly significant $(P<0.0001)$ inhibition of tumor growth was detected following 4 injections ( 3 days apart) of miR-34a formulated in NLE particles in SKMM1 xenografts (Fig. 5A). Importantly, after 21 days, we observed complete tumor regression in $50 \%$ of mice treated with formulated miR-34a mimics. Furthermore, we observed a dramatic prolongation of survival $(P=0.0009)$ of mice treated with miR-34a mimics compared with control groups, with 3 mice still surviving at 6 months (180 days) when our observation ended (median survival of
miR-34a treated group was 135 days vs. 23 days in miRNC group; Fig. 5B). Because we did not find any difference among control groups, only scrambled oligonucleotides formulated with NLE particles were used in subsequent experiments. Moreover, we also found a significant antitumor effect by intratumoral injection of formulated miR-34a in RPMI-8226 xenografts $(P=0.037$; Supplementary Fig. S6). Therefore, we conclude that miR-34a by intratumoral delivery is highly effective against TP53mutated MM xenografts and significantly prolongs host survival.

\section{Systemic delivery of formulated miR-34a inhibits growth of MM xenografts in SCID mice}

We next explored the systemic delivery potential of formulated miR34a mimics in controlling the growth of MM xenografts. We observed significant tumor growth inhibition $(P<0.01)$ in mice treated with miR-34a mimics versus controls (Fig. 5C), and this effect was associated with prolonged survival ( $P=0.041$; median survival of miR34 a treated group was 44 days vs. 26 days in miR-NC group; Fig. 5D). Interestingly, $60 \%$ of miR-34a-treated mice were still alive at the end of observation. q-RT-PCR analysis of miR-34a levels in excised tumors showed 4-fold increase of miR-34a levels (Supplementary Fig. S5B). There were large areas of necrosis with abundant nuclear debris ("dustlike" nuclear fragments, Supplementary Fig. S7A) in miR34a-treated xenografts. Moreover, MM cells exhibited cleaved caspase-3 and lower Ki-67 expression, indicating that miR-34a treatment induced inhibition of proliferation 


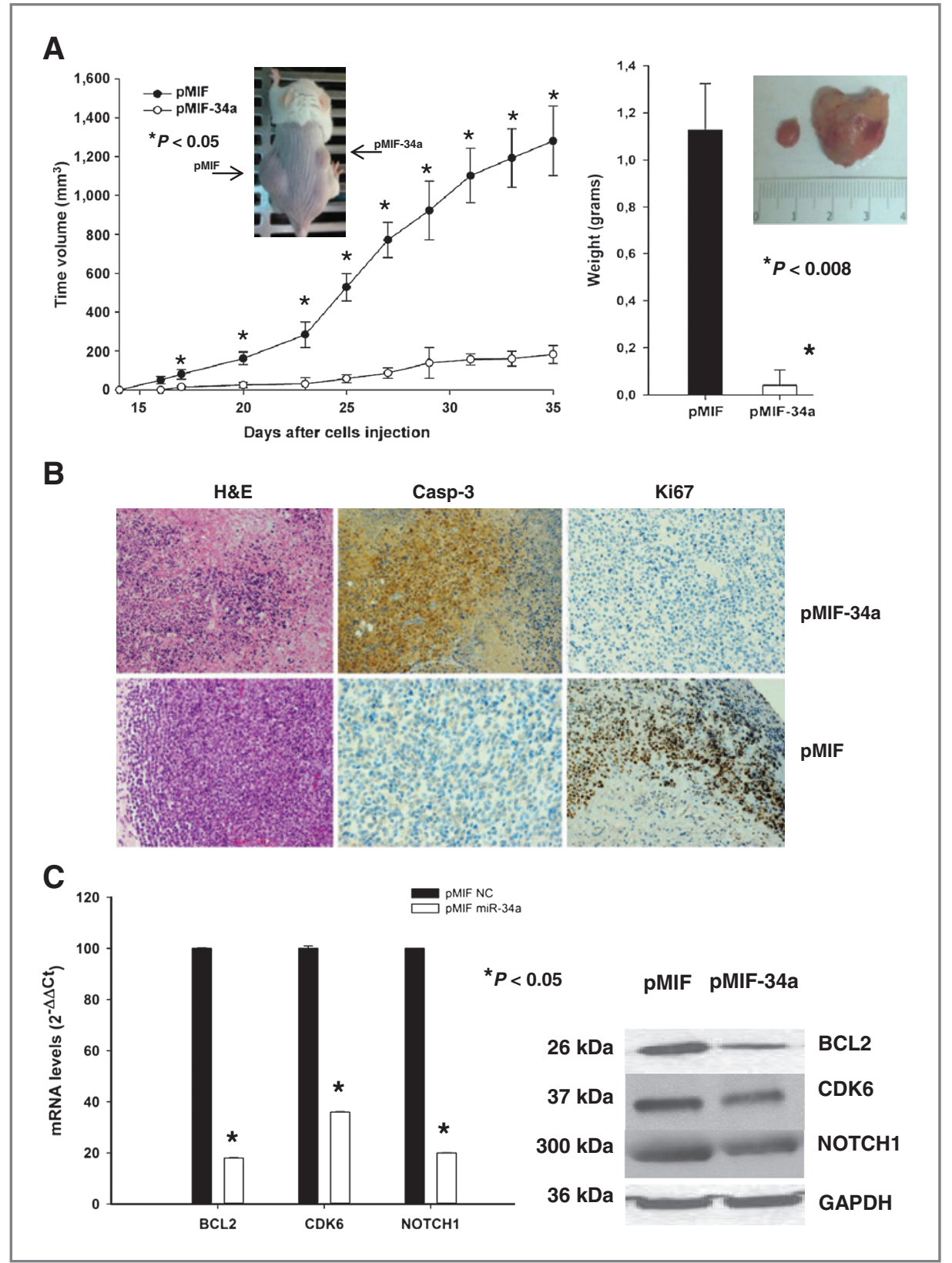

Figure 4. In vivo activity of miR-34a stably expressed in MM cells. A, in vivo tumor formation of miR-34a stably expressed in MM xenografts. Tumor volumes were measured starting day 14 after cell injection $\left(5 \times 10^{6}\right.$ pMIF-34a-SKMM1 in the right flank or pMIF-SKMM1 in the left flank) in a cohort of 10 CB-17 SCID mice. Following the detection of tumors, measurements were assessed by an electronic caliper in 2 dimensions every 2 to 3 days until the date of sacrifice or death of the first animal. The tumor volume was calculated as detailed in Materials and Methods. A representative mouse image is inserted in the graph. Tumor weight averages between pMIF-34a-SKMM1 or pMIF-SKMM1 xenografts retrieved from animals at the end of experiments show a significant difference between miR-34a transduced tumors versus controls $(P=0.008$; right). Means \pm SD weight in grams are shown. $P$ value was calculated by Student $t$ test, 2-tailed, of pMIF-34a versus pMIF tumor volumes. A representative image (insert) of a miR-34a retrieved tumor (left flank) versus control (right flank) is shown. $\mathrm{B}$, histologies and immunohistochemistry staining directed against Ki-67 and caspase-3 in pMIF-34a-SKMM1 or pMIF-SKMM1 tumors. Histologic and immunohistochemistry micrographs are at 20-fold magnification hematoxylin and eosin (H\&E) and 40-fold magnification (Casp-3 and Ki-67), respectively. C, quantitative analysis of BCL2, CDK6, and NOTCH1 mRNA (left) and protein (right) levels in retrieved pMIF-34a-SKMM1 or pMIF-SKMM1 tumors. The mRNA expression levels are shown as average after normalization with GAPDH and $\triangle \Delta \mathrm{Ct}$ calculations. Western blot analysis in retrieved tumors was conducted as described in Supplementary Methods. The protein loading control was GAPDH. Experiments were conducted in triplicate. miR-34a effects on protein levels reached statistical significance $(P<0.05)$.

and triggered apoptosis in MM xenografts in vivo. Moreover, downregulation of BCL2, CDK6, and NOTCH1 at mRNA (Supplementary Fig. S7B) and protein level (Supplemen- tary Fig. S7C) were detected by q-RT-PCR and Western blotting, respectively. Notably, analysis of treated versus control mice did not show any significant behavioral 


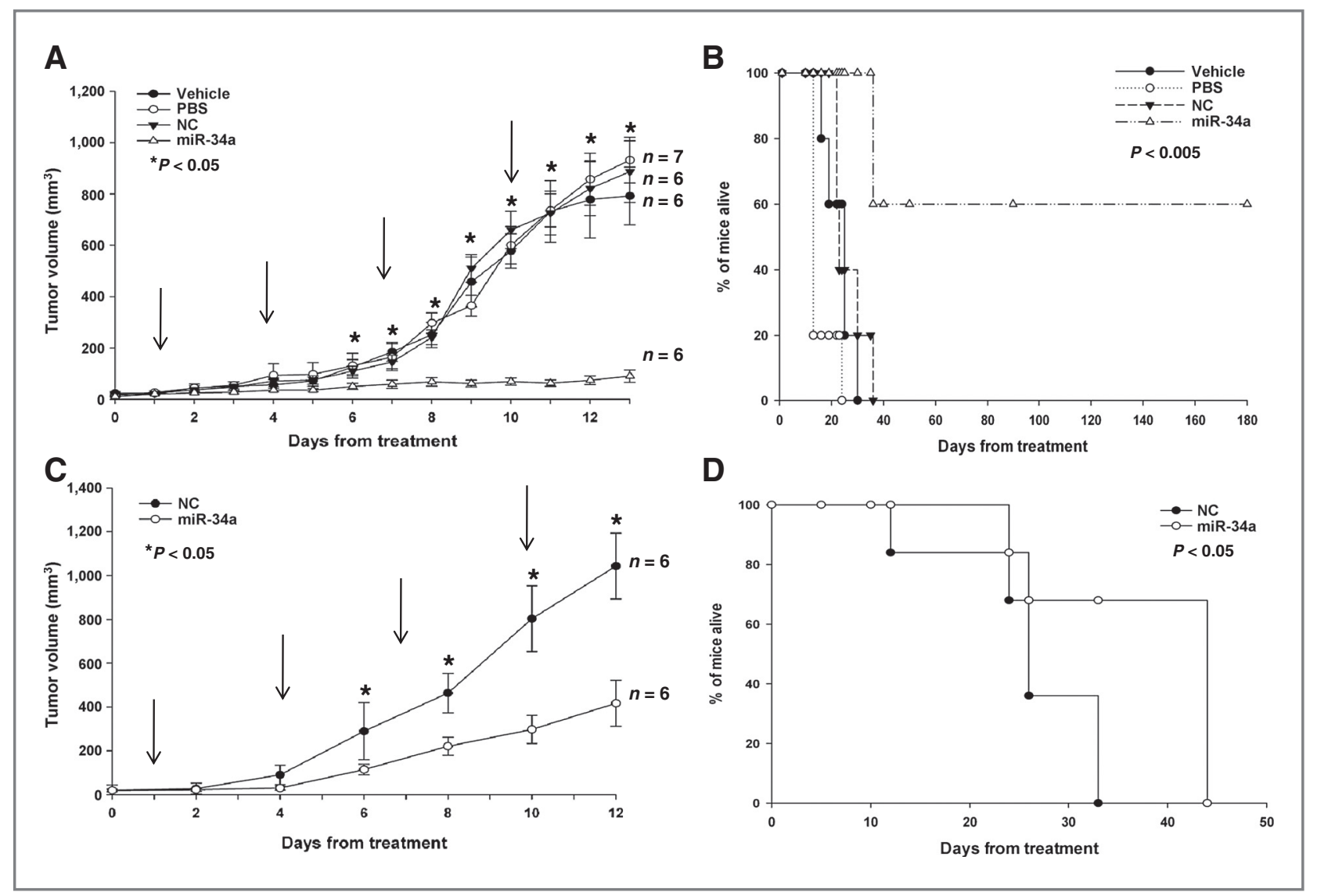

Figure 5. Intratumoral injection and systemic delivery of NLE-formulated miR-34a inhibits tumor growth in MM xenografts in SCID mice. A, effects of formulated miR-34a in SKMM1 xenografts by intratumoral injections. Palpable subcutaneous tumor xenografts were repeatedly treated every 3 days, as indicated by arrows, with $20 \mu \mathrm{g}$ of formulated miR-34a or miR-NC (NC). As control, 2 separate groups of tumor-bearing animals were injected with vehicle alone (MaxSuppressor In Vivo RNA-LANCEr II) or PBS. Tumors were measured with an electronic caliper every day, averaged tumor volume \pm SD of each group is shown. $P$ values were calculated of miR-34a versus miR-NC (Student $t$ test, 2 -tailed). ${ }^{*}$ indicates significant $P$ values $(P<0.05)$. B, survival curves (Kaplan-Meier) of intratumorally treated mice show prolongation of survival in miR-34a-treated SKMM1 xenografts compared with controls (log-rank test, $P=0.0009$ ). Survival was evaluated from the first day of treatment until death or sacrifice. Percentage of mice alive is shown. C, mice with palpable subcutaneous SKMM1 tumor xenografts were treated with $20 \mu \mathrm{g}$ of formulated miR-34a or scrambled oligonucleotides (NC) by intravenous tail vein injections. Caliper measurement of tumors was taken every 2 days from the day of first treatment. Averaged tumor volumes $\pm \mathrm{SD}$ are reported. ${ }^{*}$ indicates significant $P$ values $(P<0.05)$. D, survival curves (Kaplan-Meier) of systemically miR-34a-treated mice show prolongation of survival compared with controls (log-rank test, $P=0.041$ ). Survival was evaluated from the first day of treatment until death or sacrifice. Percentage of mice alive is shown.

changes or weight loss in SCID mice. No pathologic changes were detected by analysis of normal tissues including heart, kidney, liver, and BM of treated mice, indicating the absence of acute toxicity induced by the use of NLE formulated miR34a mimics (data not shown). Taken together, these results showed the anti-MM potential of miR-34a mimics administered by systemic injection, similar to the inhibition of tumor formation by stable transfection of lentivirus miR34a. The observed downregulation of miR-34a validated targets at both mRNA and protein levels further supports the therapeutic potential of NLE formulated miR-34a mimics. miR-34a overcomes the human BMM-dependent
protective effect on MM cells in vitro and in vivo

To further study the therapeutic potential of our findings as a novel anti-MM treatment, we evaluated the effect of miR-34a mimics on MM cells adhering to human BMSCs.
We first tested the activity of miR-34a in cocultures in vitro where primary MM cells adhered to human MM patientderived BMSCs. We found that miR-34a MM cell electroporation overcame the supportive effect of BMSCs, as evidenced by inhibition of primary MM cell proliferation (Fig. 6A). We next investigated the anti-MM activity in vivo using our recently established novel SCID-synth-hu model (43). Specifically, we engrafted primary CD138+ cells from advanced disease to evaluate the miR-34a activity when tumor cells adhered to human BMSCs. Histologic and immunohistochemical analysis of retrieved 3-dimensional biopolymeric scaffolds after treatment with formulated miR-34a showed reduced tumor infiltration and an increase of cleaved caspase- 3 and reduction of Ki-67 expression (Fig. $6 \mathrm{~B})$. These findings indicate that miR-34a overcomes the protective role of human BMM, providing an additional rationale for development of miR-34a in early clinical trials. 


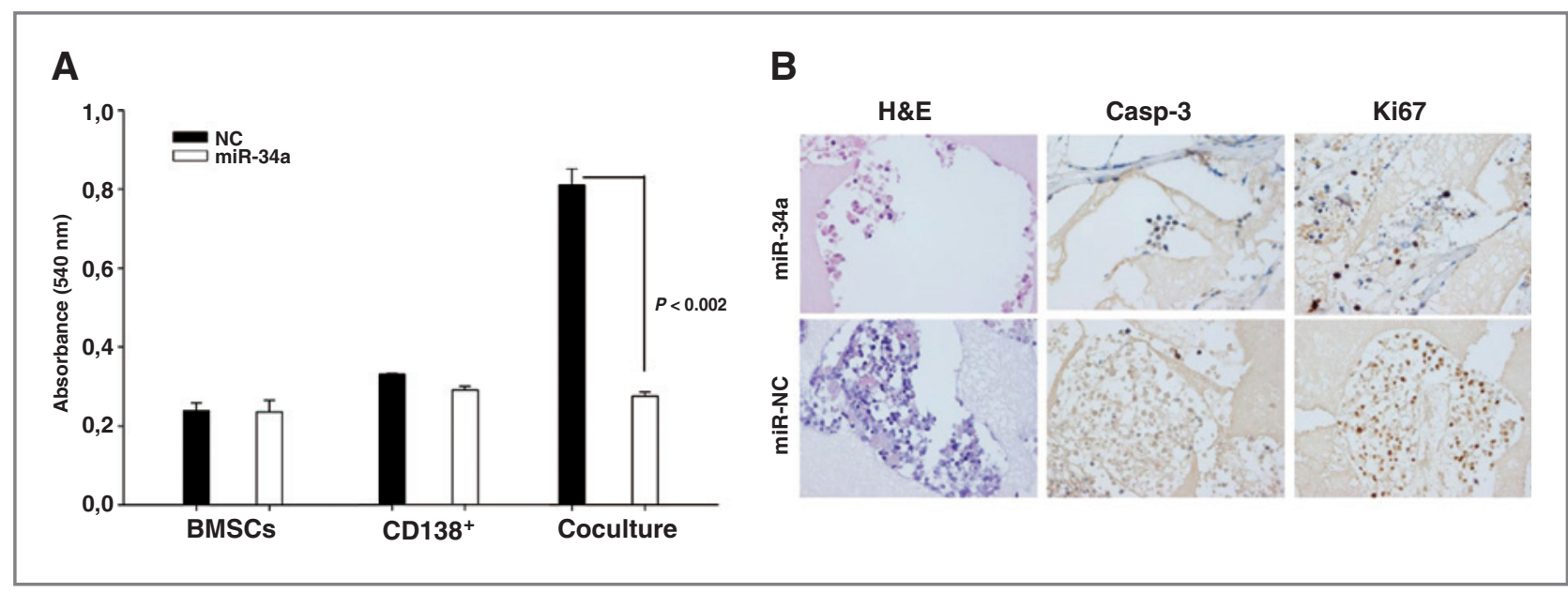

Figure 6. miR-34a exerts anti-MM activity in cocultures of primary MM-BMSCs and in the SCID-synth-hu model. A, MTS assay conducted in CD138+ cells from a MM patient cocultured with BMSCs, 24 hours after electroporation with synthetic miR-34a or miR-NC. Absorbance measurements \pm SD of 3 independent experiments are shown; significant reduction in cell survival was observed $(P<0.002$, by Student $t$ test, 2 -tailed) in cocultures treated with miR-34a. B, in vivo analysis of miR-34a in the SCID-synth-hu model. TP53-mutated primary CD138+ MM cells were injected into 3-dimensional biopolymeric scaffolds with BMSCs. Engrafted synthetic scaffolds were directly injected in vivo by NLE formulated miR-34a or miR-NC in 3 mice for each group. A representative hematoxylin and eosin (H\&E) and immunohistochemistry staining of Ki-67 and caspase- 3 on retrieved scaffolds from treated animals is shown (magnification $\times 40$ ).

\section{Discussion}

In this report, we show that synthetic miR-34a exerts a powerful antitumor activity in clinically relevant xenograft models of human MM. In vivo results were complemented by in vitro experiments where miR-34a mimics showed significant antiproliferative activity, apoptotic effects, and modulation of gene expression. To our knowledge, this is the first experimental evidence of antitumor activity of miR34a in preclinical models of MM.

An important fallout of our work is the successful delivery of miR-34a mimics in MM xenografts in SCID mice and in the SCID-synth-hu model via NLE, a novel lipid-based delivery vehicle which overcomes many of the most important limitations of other vehicles (34). In our study, the NLE-formulated miR-34a was safely administered to animals by either intratumor or systemic injection. In the latter case, the achievement of tumor growth inhibition in subcutaneous xenografts is substantial and particularly relevant, given the poor vascularization of rapidly growing xenografts. Importantly, these findings indicate the optimal bioavailability of NLE-formulated miR-34a mimics. Moreover, the miR-34a target downregulation in tumors excised from animals treated systemically with miR-34a mimics further confirms successful miRNA delivery. To provide the framework for clinical translation of this experimental approach, we further confirmed the in vivo activity of miR-34a mimics using the innovative SCID-synth- $h u$ model of human MM (43-45). In this biosynthetic and orthotopic model of human MM, tumor cells grow within a bone-like 3-dimensional biopolymeric scaffold previously engrafted with MM patient-derived human BMSCs. In this model, delivery of systemic miR-34a mimics induced significant antitumor effects, as showed by immunohistochemical analysis of retrieved scaffolds, corroborating the results obtained with the other 2 murine xenograft models of human MM used in this study. Most importantly, the antitumor properties of miR-34a mimics were not attenuated by the protective role of BMSCs, further highlighting the potential for clinical translation of our findings. In addition, the anti-MM activity of miR-34a occurred without any evidence of toxicity in mice, suggesting a favorable therapeutic index. Our data are in agreement with reports by Wiggins and colleagues (33) on the safe use of formulated NLE-miR-34a in experimental animals, and strongly support clinical development of miR-34a-based strategies in MM patients. Notably, formulated miRNA mimics are distinct from molecularly targeted drugs, as their antitumor activity relies on the modulation of a wide range of genes rather than inhibition of individual gene products. In particular, miRNA-based therapeutics can be relevant both for safety issues and to abrogate late onset of resistance, because of the complexity of miRNA-targeted pathways and the consequent low chance of developing individual "escape" mutations in the treated cells.

The molecular mechanisms of antimyeloma activity of miR34a mimics are presently under investigation. Functionally, miR-34a is a component of the TP53 transcriptional network; in TP53-wild-type cells, it is involved in a feedback loop where TP53 activates miR-34a expression, which in turn increases the activity of TP53 (31). In fact, loss of miR-34a is associated with resistance to apoptosis induced by TP53-activating agents $(21,24)$. The tumor suppressor activity of miR-34a observed in this study seems to be TP53-dependent, because it mainly occurs in tumors bearing TP53-mutated gene. In this context, it is of great interest that we found high in vivo activity of miR-34a against SKMM1 xenografts with a TP53 inactivating 
mutation but intermediate levels of miR-34a, suggesting that the anti-MM potential of miR-34a is because of more than just simple miRNA replacement in fully depleted cells. Moreover, our finding that anti-miR-34a oligonucleotide transfection in TP53 wild-type MM cells produced a growth stimulus provides further evidence of the miR-34a role as a negative regulator of $\mathrm{MM}$ cell growth and highlights the rationale of our experimental strategy of inducing miR-34a expression in TP53 mutated MM. The high sensitivity of TP53-mutated MM cells is of interest, as TP53 inactivation occurs when MM progresses to a drug-resistant and more aggressive phenotype. Although 13\% of MM patients carry TP53 coding mutations or 17q13.1 deletion causing allelic loss of TP53, 24\% of plasma cell leukemia (PCL) patients have TP53 coding mutations and 50\% of primary PCL patients or $75 \%$ of secondary PCL patients have $17 \mathrm{q} 13.1$ deletion (48-51). Furthermore, a biallelic inactivation with both coding mutation and allelic deletion has been found in $11 \%$ and $33 \%$ of primary or secondary PCL patients, respectively $(48,50,52)$, suggesting that the "biologically end-stage" disease might benefit from therapies restoring the TP53 function through miR-34a enforcement.

Of major relevance at this point is the development of therapeutic rationally designed combinations based on the study of the molecular mechanism of miR-34a activity. An interesting combination may be with gamma-secretase inhibitors taking in account NOTCH1 as relevant miR34a target. The molecular complexity of human MM highlights the need of novel miRNA-based therapeutic combinations, and the present study along with previous findings $(17,18)$, provides the basis for these research perspectives. In conclusion, the successful delivery and the antitumor activity of miR-34a mimics in clinically relevant mouse models, together with the favorable safety profile, provide

\section{Reference}

1. Anderson KC, Carrasco RD. Pathogenesis of myeloma. Annu Rev Pathol 2011;6:249-74.

2. Lonial S, Mitsiades CS, Richardson PG. Treatment options for relapsed and refractory multiple myeloma. Clin Cancer Res 2011; 17:1264-77.

3. Rajkumar SV. Treatment of multiple myeloma. Nat Rev Clin Oncol 2011;8:479-91.

4. Morgan GJ, Walker BA, Davies FE. The genetic architecture of multiple myeloma. Nat Rev Cancer 2012;12:335-48.

5. Tassone P, Tagliaferri P, Rossi M, Gaspari M, Terracciano R, Venuta S. Genetics and molecular profiling of multiple myeloma: novel tools for clinical management? Eur J Cancer 2006;42:1530-8.

6. Rossi M, Di Martino MT, Morelli E, Leotta M, Rizzo A, Grimaldi A, et al. Molecular targets for the treatment of multiple myeloma. Curr Cancer Drug Targets 2012;12:757-67.

7. Bartel DP. MicroRNAs: genomics, biogenesis, mechanism, and function. Cell 2004;116:281-97.

8. Friedman RC, Farh KK, Burge CB, Bartel DP. Most mammalian mRNAs are conserved targets of microRNAs. Genome Res 2009; 19:92-105.

9. Calin GA, Sevignani C, Dumitru CD, Hyslop T, Noch E, Yendamuri S, et al. Human microRNA genes are frequently located at fragile sites and genomic regions involved in cancers. Proc Natl Acad Sci U S A 2004;101:2999-3004. the rationale and the framework for clinical development of synthetic miR-34a mimics to improve patient outcome in MM.

\section{Disclosure of Potential Conflicts of Interest}

K.C. Anderson is an American Cancer Society Clinical Research Professor. No potential conflicts of interest were disclosed by the other authors.

\section{Authors' Contributions}

Conception and design: M.T. Di Martino, M. Ferrarini, P. Tagliaferri, P. Tassone

Development of methodology: M.T. Di Martino, M. Negrini, P. Tagliaferri, P. Tassone

Acquisition of data (provided animals, acquired and managed patients, provided facilities, etc.): M.T. Di Martino, E. Leone, N. Amodio, U. Foresta M.R. Pitari, M.E.G. Cantafio, A. Gullà, E. Morelli

Analysis and interpretation of data (e.g., statistical analysis, biostatistics, computational analysis): M.T. Di Martino, E. Leone, N. Amodio, M. Lionetti, F. Conforti, M. Ferrarini, M. Caraglia, M.A. Shammas, P. Tagliaferri, P. Tassone

Writing, review, and/or revision of the manuscript: M.T. Di Martino, M. Ferrarini, M. Caraglia, N.C. Munshi, K.C. Anderson, A. Neri, P. Tagliaferri, P. Tassone

Administrative, technical, or material support (i.e., reporting or organizing data, constructing databases): $\mathrm{V}$. Tomaino, $\mathrm{M}$. Rossi

Study supervision: P. Tagliaferri, P. Tassone

\section{Grant Support}

This work has been supported by funds of Italian Association for Cancer Research (AIRC), PI: PT. "Special Program Molecular Clinical Oncology- 5 per mille" n. 9980, 2010/15

The Editor-in-Chief of Clinical Cancer Research is an author of this article. In keeping with the AACR's Editorial Policy, a member of the AACR's Publications Committee had the article reviewed independently of the journal's review process and made the decision concerning acceptability.

The costs of publication of this article were defrayed in part by the payment of page charges. This article must therefore be hereby marked advertisement in accordance with 18 U.S.C. Section 1734 solely to indicate this fact.

Received May 30, 2012; revised August 20, 2012; accepted September 21, 2012; published OnlineFirst October 3, 2012.

10. Croce CM. Causes and consequences of microRNA dysregulation in cancer. Nat Rev Genet 2009;10:704-14.

11. Esquela-Kerscher A, Slack FJ. Oncomirs - microRNAs with a role in cancer. Nat Rev Cancer 2006;6:259-69.

12. Garzon R, Marcucci G, Croce CM. Targeting microRNAs in cancer: rationale, strategies and challenges. Nat Rev Drug Discov 2010;9: 775-89.

13. Pichiorri F, De Luca L, Aqeilan RI. MicroRNAs: new players in multiple myeloma. Frontiers in genetics 2011;2:22.

14. Lionetti M, Agnelli L, Lombardi L, Tassone P, Neri A. MicroRNAs in the pathobiology of multiple myeloma. Curr Cancer Drug Targets 2012; 12:823-37.

15. Benetatos L, Vartholomatos G. Deregulated microRNAs in multiple myeloma. Cancer 2012;118:878-87.

16. Tagliaferri P, Rossi M, Di Martino MT, Amodio N, Leone E, Gulla A, et al. Promises and challenges of microRNA-based treatment of multiple myeloma. Curr Cancer Drug Targets 2012;12:838-46.

17. Roccaro AM, Sacco A, Thompson B, Leleu X, Azab AK, Azab F, et al. MicroRNAs $15 \mathrm{a}$ and 16 regulate tumor proliferation in multiple myeloma. Blood 2009;113:6669-80.

18. Pichiorri F, Suh SS, Rocci A, De Luca L, Taccioli C, Santhanam R, et al. Downregulation of p53-inducible microRNAs 192, 194, and 215 impairs the p53/MDM2 autoregulatory loop in multiple myeloma development. Cancer Cell 2010;18:367-81. 
19. Bader AG. miR-34 - a microRNA replacement therapy is headed to the clinic. Frontiers Genet 2012;3:120.

20. Lodygin D, Tarasov V, Epanchintsev A, Berking C, Knyazeva T, Korner $\mathrm{H}$, et al. Inactivation of miR-34a by aberrant $\mathrm{CpG}$ methylation in multiple types of cancer. Cell Cycle 2008;7:2591-600.

21. Hermeking $\mathrm{H}$. The miR-34 family in cancer and apoptosis. Cell Death Differ 2010;17:193-9.

22. Chim CS, Wong KY, Qi Y, Loong F, Lam WL, Wong LG, et al. Epigenetic inactivation of the miR-34a in hematological malignancies. Carcinogenesis 2010;31:745-50.

23. Welch $\mathrm{C}$, Chen $\mathrm{Y}$, Stallings RL. MicroRNA-34a functions as a potential tumor suppressor by inducing apoptosis in neuroblastoma cells. Oncogene 2007;26:5017-22.

24. Bommer GT, Gerin I, Feng Y, Kaczorowski AJ, Kuick R, Love RE, et al. p53-mediated activation of miRNA34 candidate tumor-suppressor genes. Curr Biol 2007;17:1298-307.

25. Chang TC, Wentzel EA, Kent OA, Ramachandran K, Mullendore M, Lee $\mathrm{KH}$, et al. Transactivation of miR-34a by p53 broadly influences gene expression and promotes apoptosis. Mol Cell 2007;26: 745-52.

26. He L, He X, Lim LP, de Stanchina E, Xuan Z, Liang Y, et al. A microRNA component of the p53 tumour suppressor network. Nature 2007;447: 1130-4.

27. Tarasov V, Jung $P$, Verdoodt $B$, Lodygin $D$, Epanchintsev $A$, Menssen $A$, et al. Differential regulation of microRNAs by $p 53$ revealed by massively parallel sequencing: miR-34a is a p53 target that induces apoptosis and $\mathrm{G}_{1}$-arrest. Cell Cycle 2007;6:1586-93.

28. Tazawa $H$, Tsuchiya $N$, Izumiya $M$, Nakagama $H$. Tumor-suppressive miR-34a induces senescence-like growth arrest through modulation of the E2F pathway in human colon cancer cells. Proc Natl Acad Sci U S A 2007;104:15472-7.

29. Raver-Shapira N, Marciano E, Meiri E, Spector Y, Rosenfeld N, Moskovits $\mathrm{N}$, et al. Transcriptional activation of miR-34a contributes to p53-mediated apoptosis. Mol Cell 2007;26:731-43.

30. Ji Q, Hao X, Zhang M, Tang W, Yang M, Li L, et al. MicroRNA miR-34 inhibits human pancreatic cancer tumor-initiating cells. PLoS One 2009;4:e6816.

31. Yamakuchi M, Lowenstein CJ. MiR-34, SIRT1 and p53: the feedback loop. Cell Cycle 2009;8:712-5.

32. Liu C, Kelnar K, Liu B, Chen X, Calhoun-Davis T, Li H, et al. The microRNA miR-34a inhibits prostate cancer stem cells and metastasis by directly repressing CD44. Nat Med 2011;17:211-5.

33. Wiggins JF, Ruffino L, Kelnar K, Omotola M, Patrawala L, Brown D, et al. Development of a lung cancer therapeutic based on the tumor suppressor microRNA-34. Cancer Res 2010;70:5923-30.

34. Trang $P$, Wiggins JF, Daige $C L$, Cho $C$, Omotola $M$, Brown $D$, et al. Systemic delivery of tumor suppressor microRNA mimics using a neutral lipid emulsion inhibits lung tumors in mice. Mol Ther 2011;19: 1116-22.

35. Burger R, Guenther A, Bakker F, Schmalzing M, Bernand S, Baum W, et al. Gp130 and ras mediated signaling in human plasma cell line INA6: a cytokine-regulated tumor model for plasmacytoma. Hematol $\mathrm{J}$ 2001;2:42-53.

36. Tassone P, Neri P, Carrasco DR, Burger R, Goldmacher VS, Fram R, et al. A clinically relevant SCID-hu in vivo model of human multiple myeloma. Blood 2005;106:713-6.
37. Neri P, Kumar S, Fulciniti MT, Vallet S, Chhetri S, Mukherjee S, et al. Neutralizing B-cell activating factor antibody improves survival and inhibits osteoclastogenesis in a severe combined immunodeficient human multiple myeloma model. Clin Cancer Res 2007; 13:5903-9.

38. Fulciniti M, Hideshima T, Vermot-Desroches C, Pozzi S, Nanjappa $\mathrm{P}$, Shen $\mathrm{Z}$, et al. A high-affinity fully human anti-IL-6 mAb, 1339, for the treatment of multiple myeloma. Clin Cancer Res 2009;15: 7144-52.

39. Hamasaki M, Hideshima $T$, Tassone $P$, Neri $P$, Ishitsuka $K$, Yasui $H$, et al. Azaspirane (N-N-diethyl-8,8-dipropyl-2-azaspiro [4.5] decane2-propanamine) inhibits human multiple myeloma cell growth in the bone marrow milieu in vitro and in vivo. Blood 2005;105: 4470-6.

40. Peltier HJ, Latham GJ. Normalization of microRNA expression levels in quantitative RT-PCR assays: identification of suitable reference RNA targets in normal and cancerous human solid tissues. RNA 2008;14: 844-52.

41. Livak KJ, Schmittgen TD. Analysis of relative gene expression data using real-time quantitative PCR and the 2(-Delta Delta $C(T))$ method. Methods 2001;25:402-8.

42. Neri $P$, Tagliaferri $P$, Di Martino MT, Calimeri $T$, Amodio N, Bulotta $A$ et al. In vivo anti-myeloma activity and modulation of gene expression profile induced by valproic acid, a histone deacetylase inhibitor. $\mathrm{Br} J$ Haematol 2008;143:520-31.

43. Calimeri T, Battista E, Conforti F, Neri P, Di Martino MT, Rossi M, et al. A unique three-dimensional SCID-polymeric scaffold (SCID-synth-hu) model for in vivo expansion of human primary multiple myeloma cells. Leukemia 2011;25:707-11.

44. Tassone $\mathrm{P}$, Neri $\mathrm{P}$, Burger R, Di Martino MT, Leone E, Amodio N, et al. Mouse models as a translational platform for the development of new therapeutic agents in multiple myeloma. Curr Cancer Drug Targets 2012;12:814-22.

45. DeWeerdt S. Animal models: towards a myeloma mouse. Nature 2011;480:S38-9.

46. Vassilev LT, Vu BT, Graves B, Carvajal D, Podlaski F, Filipovic Z, et al. In vivo activation of the $\mathrm{p} 53$ pathway by small-molecule antagonists of MDM2. Science 2004;303:844-8.

47. Lionetti M, Neri A. Unpublished results.

48. Jimenez-Zepeda VH, Dominguez-Martinez VJ. Plasma cell leukemia: a highly aggressive monoclonal gammopathy with a very poor prognosis. Int J Hematol 2009;89:259-68.

49. Tiedemann RE, Gonzalez-Paz N, Kyle RA, Santana-Davila R, PriceTroska T, Van Wier SA, et al. Genetic aberrations and survival in plasma cell leukemia. Leukemia 2008;22:1044-52.

50. Lloveras E, Granada I, Zamora L, Espinet B, Florensa L, Besses C, et al Cytogenetic and fluorescence in situ hybridization studies in 60 patients with multiple myeloma and plasma cell leukemia. Cancer Genet Cytogenet 2004;148:71-6.

51. Chng WJ, Price-Troska T, Gonzalez-Paz N, Van Wier S, Jacobus S Blood E, et al. Clinical significance of TP53 mutation in myeloma Leukemia 2007;21:582-4.

52. Gertz MA, Lacy MQ, Dispenzieri $A$, Greipp PR, Litzow MR, Henderson $\mathrm{KJ}$, et al. Clinical implications of $\mathrm{t}(11 ; 14)(\mathrm{q} 13 ; \mathrm{q} 32), \mathrm{t}(4 ; 14)(\mathrm{p} 16.3 ; \mathrm{q} 32)$, and $-17 \mathrm{p} 13$ in myeloma patients treated with high-dose therapy. Blood 2005; 106:2837-40 


\section{Clinical Cancer Research}

\section{Synthetic miR-34a Mimics as a Novel Therapeutic Agent for Multiple Myeloma: In Vitro and In Vivo Evidence}

Maria T. Di Martino, Emanuela Leone, Nicola Amodio, et al.

Clin Cancer Res 2012;18:6260-6270. Published OnlineFirst October 3, 2012.

Updated version Access the most recent version of this article at: doi:10.1158/1078-0432.CCR-12-1708

Supplementary Access the most recent supplemental material at:

Material http://clincancerres.aacrjournals.org/content/suppl/2012/10/03/1078-0432.CCR-12-1708.DC1.html

Cited articles This article cites 50 articles, 14 of which you can access for free at:

http://clincancerres.aacrjournals.org/content/18/22/6260.full.html\#ref-list-1

Citing articles This article has been cited by 11 HighWire-hosted articles. Access the articles at:

http://clincancerres.aacrjournals.org/content/18/22/6260.full.html\#related-urls

E-mail alerts Sign up to receive free email-alerts related to this article or journal.

Reprints and To order reprints of this article or to subscribe to the journal, contact the AACR Publications Department at Subscriptions pubs@aacr.org.

Permissions To request permission to re-use all or part of this article, contact the AACR Publications Department at permissions@aacr.org. 